University of Wollongong

Research Online

Faculty of Engineering and Information

Faculty of Engineering and Information

Sciences - Papers: Part B

Sciences

2018

Three-dimensional measurement of small-object surfaces on the basis of fiber optic interferometric fringe projection

Xiaojie Li

Tianjin Polytechnic University

Xiaojie Duan

Tianjin Polytechnic University

Guangxu Li

Tianjin Polytechnic University

Teng Cao

Tianjin Polytechnic University

Rui Xie

Tianjin Polytechnic University

See next page for additional authors

Follow this and additional works at: https://ro.uow.edu.au/eispapers1

Part of the Engineering Commons, and the Science and Technology Studies Commons

Research Online is the open access institutional repository for the University of Wollongong. For further information contact the UOW Library: research-pubs@uow.edu.au 


\title{
Three-dimensional measurement of small-object surfaces on the basis of fiber optic interferometric fringe projection
}

\author{
Abstract \\ We used a system that exploits the Mach-Zehnder interferometer structure and Young's double-pinhole \\ interference principle to measure the three-dimensional topographies of small objects at high precision. \\ Next, we performed phase profilometry to measure small objects and achieve a height measurement \\ within a $10 \times 10 \mathrm{~cm}$ area. The accuracy of the measurement system improved by $44.1 \%$, and the \\ measurement time was reduced by $63.2 \%$. \\ Disciplines \\ Engineering | Science and Technology Studies

\section{Publication Details} \\ Li, X., Duan, X., Li, G., Cao, T., Xie, R., Hong, Y., Tang, C., Li, E., Zhang, C. \& Li, H. (2018). Three-dimensional \\ measurement of small-object surfaces on the basis of fiber optic interferometric fringe projection. Optical \\ Engineering, 57 (9), 094105-1-094105-9.

\section{Authors} \\ Xiaojie Li, Xiaojie Duan, Guangxu Li, Teng Cao, Rui Xie, Yanhua Hong, Chunxiao Tang, Enbang Li, Cheng \\ Zhang, and Hongqiang Li
}




\title{
Three-dimensional measurement of small-object surfaces on the basis of fiber optic interferometric fringe projection
}

\author{
Xiaojie Li, ${ }^{a}$ Xiaojie Duan, ${ }^{a}$ Guangxu Li, ${ }^{a}$ Teng Cao, ${ }^{a}$ Rui Xie, ${ }^{a}$ Yanhua Hong, ${ }^{b}$ Chunxiao Tang, ${ }^{a}$ Enbang Li, ${ }^{c}$ \\ Cheng Zhang, ${ }^{a}$ and Hongqiang Lía \\ ${ }^{a}$ Tianjin Polytechnic University, School of Electronics and Information Engineering, Tianjin Key Laboratory of Optoelectronic \\ Detection Technology and Systems, Tianjin, China \\ ${ }^{\mathrm{b} B a n g o r}$ University, School of Electronic Engineering, Bangor, United Kingdom \\ 'University of Wollongong, School of Physics, Wollongong, New South Wales, Australia
}

\begin{abstract}
We used a system that exploits the Mach-Zehnder interferometer structure and Young's doublepinhole interference principle to measure the three-dimensional topographies of small objects at high precision. Next, we performed phase profilometry to measure small objects and achieve a height measurement within a $10 \times 10-\mathrm{cm}$ area. The accuracy of the measurement system improved by $44.1 \%$, and the measurement

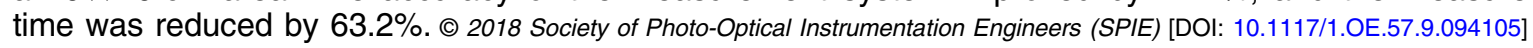

Keywords: fiber-optic interference; three-dimensional shape; small object.
\end{abstract}

Paper 180756 received May 23, 2018; accepted for publication Aug. 29, 2018; published online Sep. 14, 2018.

\section{Introduction}

Image processing, computer, and multimedia technologies are widely applied to numerous fields, such as industry, national defense, healthcare, security, and entertainment; therefore, these technologies require the three-dimensional (3-D) shape information of objects to address and display data directly. A new optical information method developed using 3-D shape measurement technology exploits an optical image to detect and transmit information and extract relevant details from captured images, thus completing the recovery and measurement of the 3-D shapes of objects in a computer $^{\mathbb{Q}}$ Therefore, phase measurement profilometry based on fringe-structured light projection satisfies the demand for 3-D object surface topography measurement in the previously mentioned application domains. This technology applies the principle of optical triangulation measurement, which can also be applied to the 3-D surface topography measurement of static and dynamic objects. $\mathrm{QB}$ However, the demand for the 3-D topography measurement of small objects is increasing, particularly in current industry applications for measurements, such as the detection of fine pits on bearings, small deformations of springs, and defects on the surfaces of battery casings. However, modern optical 3-D topography measurement techniques have certain limitations in measuring small objects. In particular, the accuracy and speed of phase extraction need to be improved. The selfadaptability and degree of automation of object shape measurement are low because the density of stripes owing to digital light processing (DLP) technology is low in small regions and is thus unsuitable for small-sized objects. Laser interference projection can dynamically control the density of interference fringes and is conducive to the integration of measurement systems. Therefore, laser interference projection is suitable for measuring the 3-D topography of small-sized objects and has a high application value and range.
The first component of the measurement system is a structured light projection device. In previous years, physical grating and mechanical equipment have been used as the main modes of achieving fringe pattern projection and phase shifting. However, the density of the projected fringes and the accuracy of the phase shift are low. In recent years, DLP has been commonly used for projecting fringe images generated by programming. The density of the fringes is limited by the projection view field and resolution of the DLP. Therefore, the DLP is unsuitable for measuring small objects. Thus, we utilized two beams of coherent light to produce the structured light patterns of interference fringes, thereby forming high-density fringe-structured light in a small field-of-view and projecting them onto the surface of the measured object. However, the structure of the fringe projection system based on the previously mentioned method is relatively complex, and the positional relationship among the optical elements considerably affects the nature of the projected fringe patterns. Moreover, the fringes are susceptible to temperature fluctuations, environmental vibrations, and other factors. Thus, the optical interference system requires stabilization control. Numerous scholars explored phase stability control of the optical interferometer. Matthews and Mercer used the PTDC (DC phase tracking) and PTAC (AC phase tracking) technologies, respectively, to achieve phase stability control of the optical interferometer ${ }^{103}$ Freschi and Frejlich ${ }^{\square}$ utilized optical high-frequency phase modulation and phase-locking technologies to enable the interferometer to function at all stable operating points. Kudryashov and Seliverstov ${ }^{\square}$ used photodiodes to detect the optical intensity of the interferometer fringe and achieve phase control by changing the output wavelength of the laser. Moore et al $\mathbb{1}^{\mathbb{6}}$ controlled the interferometer to achieve an orthogonal steady state by using a piezoelectric ceramic transducer (PZT). Wu et al. 10 used a photoelectric detection circuit to detect the light intensity 
changes of the stripes and achieve phase stability control of the interference fringes.

In this work, we focus on the high-accuracy measurement of small-object 3-D surface topography. A new 3-D shape measurement technique based on optical fiber interference fringe-structured light projection, which utilizes the MachZehnder fiber interferometer structure and Young's theory of double-hole interference, is developed. The purpose is to achieve the projection of interference fringes with high density and cosine distribution. The interference fringe images modulated by the measured object are obtained by using an array camera. Thus, preprocessing the acquired fringe images on the basis of undamaged cosine distribution feature in normal direction generates a high signal-tonoise ratio. Thus, the extraction accuracy of the fringe phase is improved. The 3-D surface shape of the measured object combined with the mathematical model of the measurement system is recovered.

Optical fiber is used as the optical transmission medium. The measurement system integration is improved by enhancing the compactness of the projection system. Unlike the commonly used methods applying DLP and physical gratings, this study utilized optical fiber interference to generate a high-density fringe structure light-image in a very small range, which exhibits remarkable spatial cosine distribution characteristics. Therefore, the small size and flexibility of the fiber probe renders this material suitable for measuring complex and small objects in tight spaces or harsh environments. This system has favorable application prospects.

\section{Optical Geometry Model of Double-Fiber-Optic Interference Fringe Projection}

The diameter of the optical fiber is minute; the diameter of this single-mode fiber is $4.3 \mu \mathrm{m}$. Thus, the optical fiber projection-end can be viewed as two associated point-lights that satisfy the conditions of optical interference. According to the principle of Young's double-hole optical interference, the brightness level of the interference fringes forms a hyperboloid family with the two projection ends as the focal points. Theoretically, a set of hyperbolic graphs form on the rear projection screen in the interference light field. However, the background screen in an actual interference system appears as a group of light-staggered and dark-staggered distribution and straight fringe graphs. In this work, we use the mathematical model of the optical fiber interferometer fringe projection system and discuss the conversion of the shape of the brightness-level fringe curve on the background screen into a linear one.
We assume that two coherent light sources emit a spherical optical wave at identical frequency and vibration direction. In the overlay area of the optical wave, the light intensity of a point in the interference field is

$I=I_{1}+I_{2}+2 \sqrt{I_{1} I_{2}} \cos (\Delta \theta)$,

where $I_{1}$ and $I_{2}$ denote separate intensities in the field of the two light sources, and $\Delta \theta$ is the corresponding phase difference. In Eq. (1), $I$ is the maximum, and the brightness fringes at this point are present when $\Delta \theta=2 k \pi$, $k=0, \pm 1, \pm 2, \ldots I$ is the minimum. The two interference lights repel each other when $\Delta \theta=(2 k+1) \pi, k=0$, $\pm 1, \pm 2 \ldots$ Dark fringes appear, and the level of interference fringes is denoted as $k$. The light intensity stabilizes in the time domain of the interference field although not in the spatial domain in the variation pattern of the cosine distribution.

A fringe-structured light projection method is designed using a double-optical-fiber interferometer. Two optical fiber output-ends are used as two light sources. Then, the interference phenomena of the output light field are investigated, and the mathematical model is established. In Fig. 11, the centers of the optical fiber outputs are denoted as $S_{1}$ and $S_{2}$ in the $x, y$, and $z$ coordinate axes system. The corresponding coordinates are $S_{1}(0, d / 2,0)$ and $S_{2}(0,-d / 2,0)$, which are in accordance with the principle of optical wave interferometry. $P$ is assumed to be a random point on a rear-observing screen (UV plane) in the optical projection field, wherein the space coordinates are $P(x, y, z)$. The optical path difference of $P$ to the optical fiber output end is

$$
\begin{aligned}
\Delta r=\left|r_{1}-r_{2}\right|= & \mid \sqrt{\left(y-\frac{d}{2}\right)^{2}+x^{2}+z^{2}} \\
& -\sqrt{\left(y+\frac{d}{2}\right)^{2}+x^{2}+z^{2}} \mid
\end{aligned}
$$

According to the principle of light wave interference, the positional relationship among all the levels of light and dark fringes in the UV plane is

$\Delta r=k \cdot \frac{\lambda}{2},(k=0, \pm 1, \pm 2, \ldots)$,

where $\lambda$ is the output wavelength of a laser. We selected the light-level fringes and obtained the spatial distribution of the light-level interferometric fringes, which are hyperboloid graphics based on the $y$-axis of the rotation axis; the spatial distribution is expressed as follows:

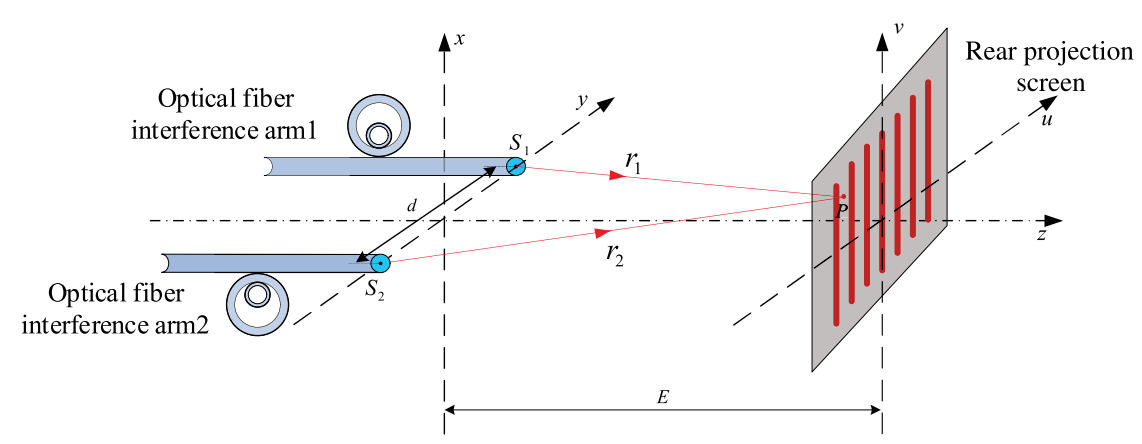

Fig. 1 Interference spatial distribution of dual-optical-fiber output field. 


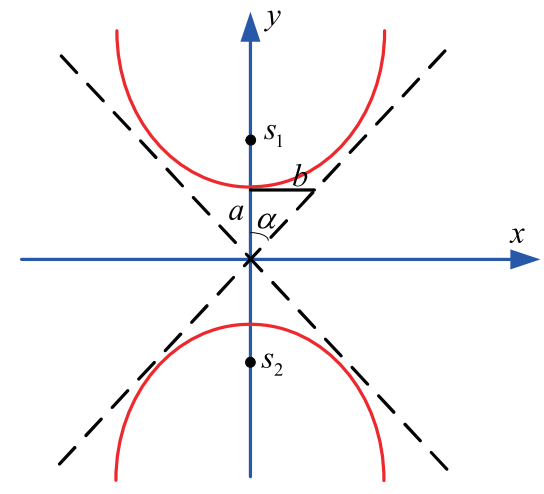

Fig. 2 Diagram of hyperbolic asymptotic line.

$$
\frac{y^{2}}{\left(\frac{k \lambda}{2}\right)^{2}}-\frac{x^{2}+z^{2}}{\left(\frac{d}{2}\right)^{2}-\left(\frac{k \lambda}{2}\right)^{2}}=1, \quad(k=0, \pm 1, \pm 2, \ldots) .
$$

A rear-observing screen is installed in the spatial distribution of the dual-optical-fiber output field. It is vertically

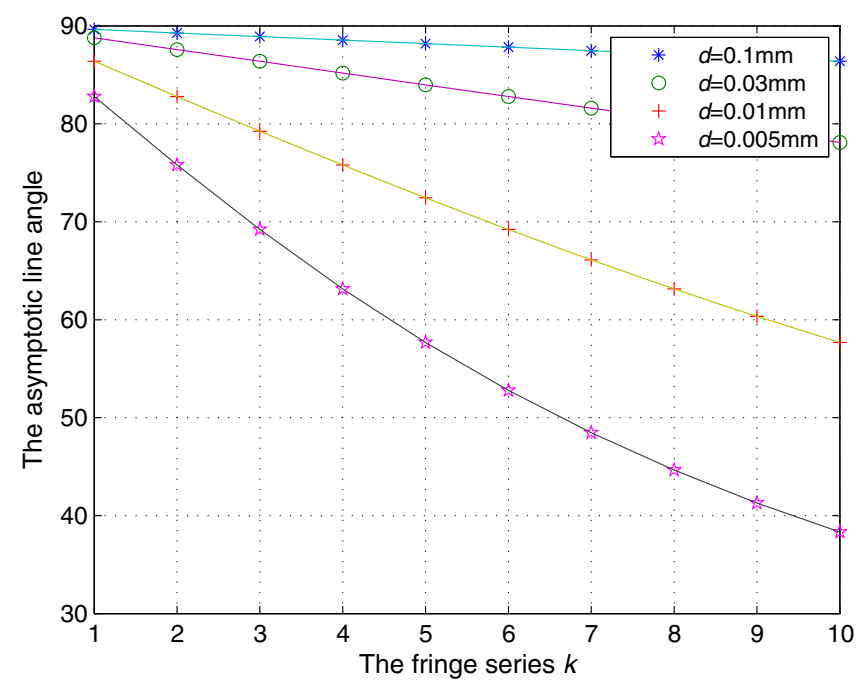

Fig. 3 Angle $\alpha$ of asymptotic line at different values of distance $d$ of fiber core.

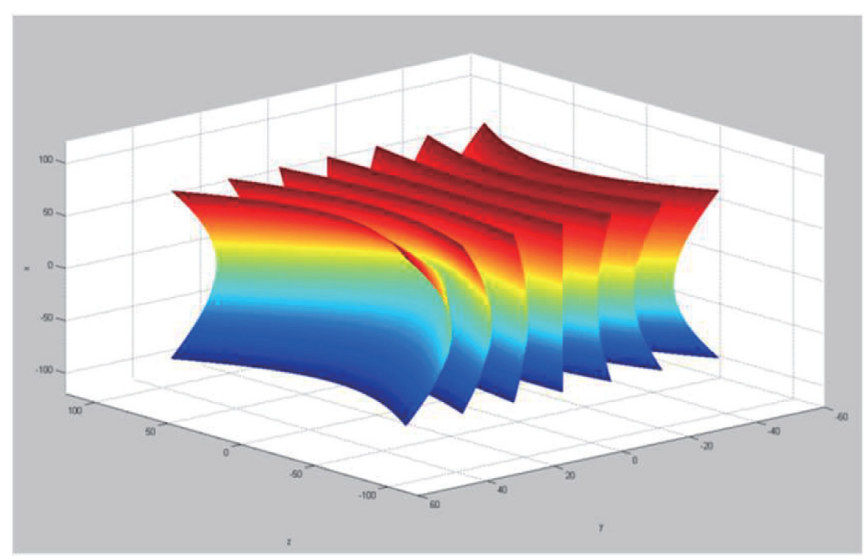

(a) generally surface mode1 directed to the $x, o$, and $z$ planes, which exhibited a set of hyperbolic light-class shape interference fringes.

The dashed line is the asymptotic line of the fringes, as shown in Fig. 2. $K$ is the slope, and $\alpha$ is the angle. If $d \gg k \lambda(k=0, \pm 1, \pm 2 \ldots), \quad \lambda=632.8 \mathrm{~nm}$ and $K \rightarrow \infty$. Therefore, we obtained $\operatorname{tg} \alpha \rightarrow \infty$; where $\alpha \approx \pi / 2$, thereby obtaining different values of the distance $d$ of fiber cores.

In Fig. 3, if the distance $d$ of the fiber core is smaller than the laser output wavelength $\lambda$, then the slope angle of the asymptotic line gradually decreases. Therefore, the asymptotic lines of all of the level fringes exhibit a cross distribution. Otherwise, if $d \gg k \lambda$, all the angles of the asymptotic lines are $\sim 90 \mathrm{deg}$. Thus, all the asymptotic lines indicate a parallel distribution.

The distance of the rear plane to the optical fiber output end is larger than the distance of the two optical fiber cores, which is referred to as the far-field condition $(z \gg d)$, when the distance of $P$ in the plane to the $z$-axis is less than that of $P$ to the midpoint of the two optical fiber core connection lines, i.e., the paraxial optic condition $\left[z_{2} \gg\left(x_{2}+y_{2}\right) \max \right]$. Therefore, a set of approximate parallel and equidistant distributions of light and dark fringe patterns is obtained when the previously mentioned two conditions are satisfied. If the distance of the plane to the fiber projection end is constant (denoted as $E$; i.e., $z=E$ ), the spatial distance of two adjacent light-class interference fringes in the $y$-direction is

$\Delta y=\frac{\lambda E}{d}$.

The distribution of the light-class interference fringe light surface model, which is shown in Fig. 4(a), can be obtained by applying the mathematical modeling and simulation to the dual-fiber interferometer fringe projection system. To achieve a clear projection fiber interference fringe, the projection space distances are set to $1000 \mathrm{~mm}$. The light surface models of the light-class fringes at $k=0, \pm 1, \pm 2, \pm 3$ are shown in Fig. 4(b).

\section{Mathematical Model of Measurement System}

The linearity of light and dark fringe optical surfaces gradually improves with the increase in the projection distance and the satisfaction of the far-field and paraxial optics conditions.

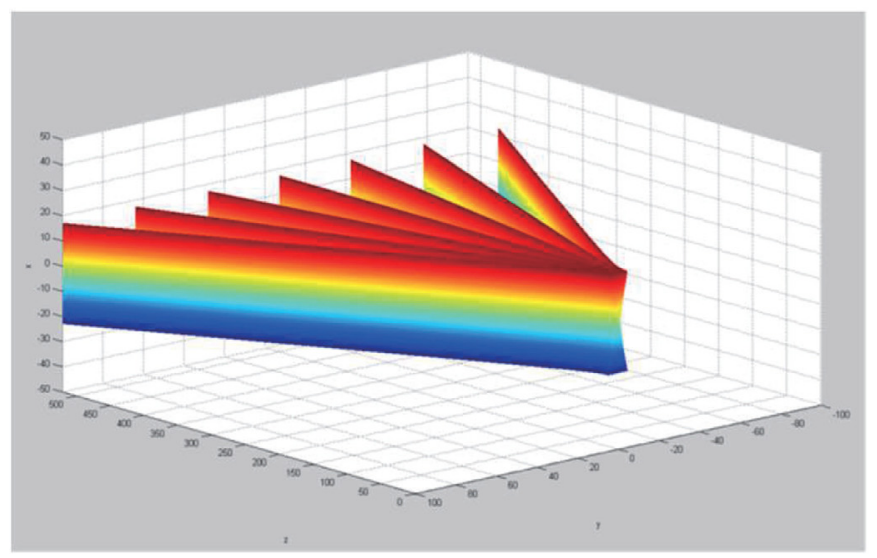

(b) projection space distances:1000mm

Fig. 4 Light surface models of light-class interference fringes at different projection distances. (a) Generally surface model and (b) projection space distances: $1000 \mathrm{~mm}$. 
The interference fringes are projected onto a plane surface. The intensity distribution of the fringes in the parallel direction to the connection line of the fiber output-end core is a cosine function.

This section focuses on the construction of the mathematical model of the relationship between the phase of the projected fringes and the surface height of the measured object. In the reference plane $R$, we assumed two orthogonal directions, namely, the $x$ - and $y$-axes. The vertical direction to $R$ is the $z$-axis, whereas the interference fringe projected in the direction parallel to $R$ is the $x$-axis. An array camera is used for capturing the fringe images in the $z$ direction. The height difference $h(x)$ deforms the interference fringes when objects are present on $R$, and the deformation quantity is relative to the height difference $h(x)$ of the measured object surface to $R$. In the fringe images, the intensity of light points with equal $y$ values is different from the cosine

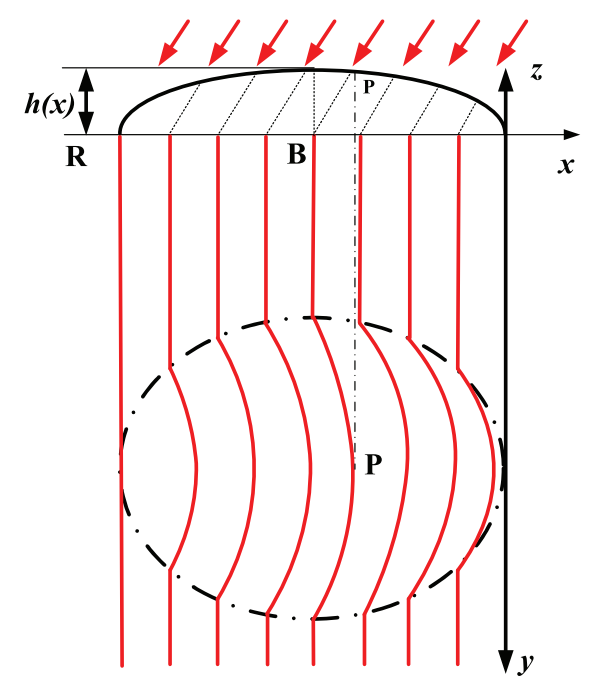

Fig. 5 The top view of fringes modulated by object. distribution in the same period, as shown in Fig. 5. All the points in the captured images are modulated by the measured objects, and the parallel fringe images of $R$ are different. The phase variation depends on the actual 3-D height (depth) of the point on the object's surface.

A mathematical model of the measured object height $h(x)$ and the modulated interference fringe-phase information $\Delta \varphi(x)$ are obtained, as shown in Fig. 6. The optical center of the plane array camera lens is $E_{c}$. The connection line center of the two optical fiber output-ends is $E_{p} . P$ is any point on the surface of the measured object. The projection of point $B$ on $R$, which is modulated by the measured object, becomes $P$ when an object is placed before $R$. The projection of $P$ on $R$ is $P^{\prime}$. Therefore, $P P^{\prime}$ is the height $h(x)$ of point $P$.

The point $B$ is likely to shift to the point $A$ in the interference fringe images captured by the plane array camera because the light intensity at $A$, which is similar to that at $P$, on the reference plane is modulated by the object surface.

Therefore, the height of $P$ is related to the distance of $A$ that shifts to point $B$. The mathematical model of the measurement system is derived from the fixed $y$-axis. In accordance with the similar triangle relationship of $E_{p} P E_{c}$ with $\mathrm{APB}$, the following expression is obtained:

$\frac{\left|x_{A}-x_{B}\right|}{d_{0}}=\frac{|h(x)|}{l_{0}-|h(x)|}$,

where $d_{0}$ is the distance of the fiber output-end cores to the camera lens center and referred to as the baseline distance. The height of the camera lens optical center to the reference plane is $l_{0}$. Then, the interference fringe images of the cosine distribution are projected onto the reference plane. In the captured image $s(x)$ with spatial frequency $f_{0}$ in the $x$-direction, $A$ is the background light intensity of the interference fringe images. The projected optic intensity $s(x)$ on the reference plane in the $x$-direction is

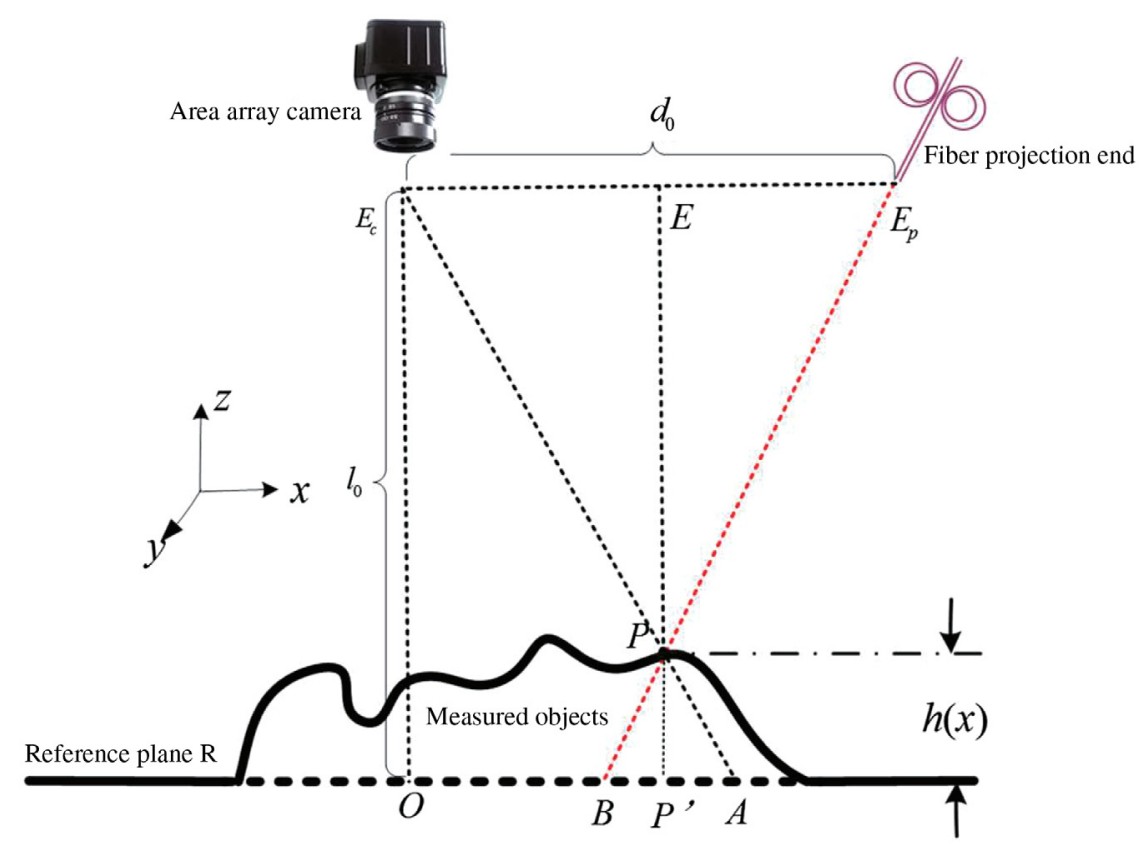

Fig. 6 Phase-height geometry model of the measured object. 


$$
s(x)=A \cos \left[2 \pi f_{0} x+\varphi(x)\right]
$$

In the reference plane $R$, the point $B$ shifted to the point $A$, which corresponds to shift of phase $\varphi_{B}(x)$ to $\varphi_{A}(x)$. Therefore, $\quad A B=\left|x_{A}-x_{B}\right|=\Delta \varphi(x) / 2 \pi f_{0} \quad$ is derived. Hence, the height of point $P$ is

$$
|h(x)|=\frac{l_{0} \Delta \varphi(x)}{2 \pi f_{0} d_{0}+\Delta \varphi(x)} .
$$

In the equation $\Delta \varphi(x)=\varphi_{A}(x)-\varphi_{B}(x)$, the full-field 3-D information of the object surface topography can be obtained by utilizing the array camera for capturing the deformation fringe images modulated by a measured object and the images of a reference plane. Then, a digital image processing method is used for obtaining the entire viewfield phase change.

\section{Stabilization Control of Phase of Interference Fringes}

The system uses the optical interferometer to design a fringestructured light projection system. The main factors that cause the phase shift of the interference fringe patterns on the screen in the projected light field are as follows: (1) stability of the laser output wavelength, (2) change in the length and refractive index of the fiber caused by the temperature variation, (3) polarization changes in the optical fiber light, and (4) structured light projection distance variation caused by the environmental vibration. The wavelength stability is determined in the experiment, and the polarization changes are not the main factors affecting the phase of the interference fringes. The projection system is constructed on a precision optical vibration isolation platform, which strongly inhibits the projection distance changes caused by the environmental vibration. Therefore, the optical path difference and refractive index changes of the fiber interference arm caused by the ambient temperature fluctuations are the main factors that stabilize the fringe phases.

If the laser is placed in the optical fiber and the length of the fiber is $L$, the variations in the fiber refractive index and length is likely to cause the projected fringe phase change $\Delta \varphi$

$\Delta \varphi=\frac{2 \pi}{\lambda} L\left(T_{0}\right) n\left(T_{0}\right)\left(\alpha_{1}+C_{T}\right)\left(T-T_{0}\right)$, where $L$ is the fiber length when the temperature is $T_{0}, n\left(T_{0}\right)$ is the refractive index at different temperatures $(n \approx 1.46$, $T_{0}=25^{\circ} \mathrm{C}$ ), and $\alpha_{1}$ is the coefficient of linear thermal expansion when the wavelength of the laser is $632.8 \mathrm{~nm}$. The temperature coefficient of optical refraction is $C_{T} \approx 0.662 \times 10-5$. The length difference between the two fiber arms in the interference fringe projecting system was 20 to $30 \mathrm{~mm}$. A phase shift of 2 to $3 \mathrm{rad}$ is obtained in Eq. (9), and the ambient temperature change is $10^{\circ} \mathrm{C}$. Thus, the phase shift affects the accuracy of the 3-D morphology measurement of a tested object based on the optical fiber-projected fringes. The phase stability control of the interference fringes is highly critical.

The phase of the projected fringes should remain stable such that the images captured by a camera are accurate and clear. The phase shift of the interference fringes can be achieved by adjusting the laser output wavelength and position of the background viewing screen. However, the following two major disadvantages are identified: (1) The change in the output wavelength of the laser would generate noise, and the laser spectral linewidth could affect the projected fringe contrast. (2) The phase requires precision automatic displacement equipment to change the position of the viewing screen and establish various projection distances.

In this work, the fiber arm length is changed through the proposed method for the stable control of the interference fringes. Furthermore, an optical phase control system is used to modulate the fiber arm lengths in accordance with the optical path difference and for stabilizing the projected fringe phase. The optical fiber interferometer arm is intertwined with the cylindrical PZT. The optical phase modulation system generates the control signal to deform the PZT. The fiber arms are stretched and compressed, thereby changing the optical path difference of the two interference arms to offset the phase shift caused by the ambient temperature fluctuation and to stabilize the projected fringe phase.

Figure 7 shows an all-fiber model for an optical interferometer, which has numerous advantages as follows: (1) only a pair of the coupling lens is required for reducing the optical power losses in coupling. (2) The Fresnel reflection light signal at the output terminal of the two interferometer arms disrupts the output terminal of the $2 \times 23-\mathrm{dB}$ optical couple when the Michelson optical fiber interferometer is used. The reflected interference optical signal can be used for monitoring the phase difference between the two interferometer arms and achieve a closed-loop phase control through the

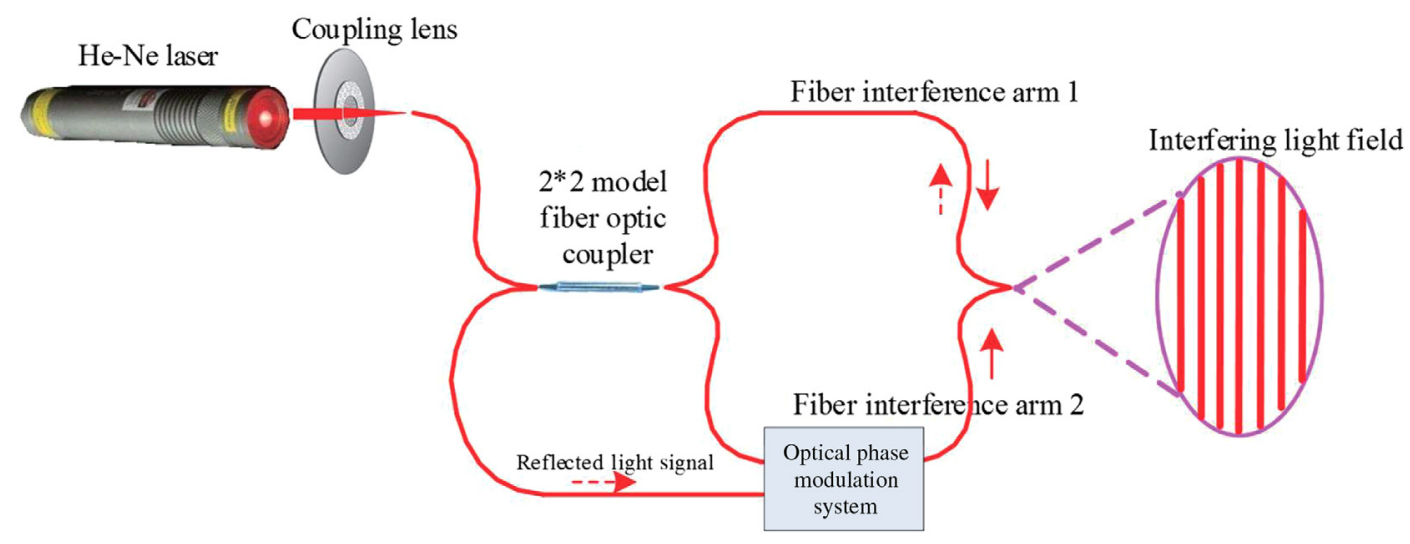

Fig. 7 Mach-Zehnder fiber interferometer phase modulation chart. 
optical phase modulation system. For laser transmission in a single-mode fiber, the phase information of which is straightforwardly affected by the temperature fluctuations in the environment, a fringe phase control system is designed, which utilizes the PZT as the strain drive sensor. The axial strain alters the optical path, which compensates for the interference caused by the fringe phase shift. A phasetracking algorithm is used to monitor the phase fluctuation of the interferometer arms. Then, a servo control system is designed for producing a control signal required for driving the PZT and for controlling the phase stability of the interference fringes.

The principle of the active phase-tracking homodyne detection method based on the optical fiber interferometer is as follows: the phase-tracking detection and modulation units produce identical phase variation and are converted to a voltage signal when the temperature fluctuations cause the phase shift of the interference fringes. Therefore, the phase stability control is compensated by the amount of phase shift.

The phase-tracking detection and modulation unit is installed on the optical fiber interferometer arm to achieve the phase detection and modulation of the interferometer arm by the feedback voltage signal, thereby validating the effectiveness of the phase difference between the two interferometer arms in the quadrature condition

$$
\varphi_{0}+\varphi_{s}(t)-\Delta \varphi(t)=n \pi+\frac{\pi}{2} n=0, \pm 1, \pm 2, \ldots,
$$

where $\varphi_{0}$ is the initial phase difference between the interferometer arms, $\varphi_{s}(t)$ is the random phase variation of the interferometer arms caused by environmental temperature fluctuations, and $\Delta \varphi(t)$ is the phase shift compensation of the phase-tracking modulation units. The output voltage of the phase detection unit that detects the light intensity from the two optical fiber arm ends can be expressed as follows:

$$
\left\{\begin{array}{l}
V_{1}(t)=V\left\{1+\frac{1}{2} \cos \left[\varphi_{0}+\varphi_{s}(t)-\Delta \varphi(t)\right]\right\} \\
V_{2}(t)=V\left\{1-\frac{1}{2} \cos \left[\varphi_{0}+\varphi_{s}(t)-\Delta \varphi(t)\right]\right\}
\end{array} .\right.
$$

After the differential amplifier, $V_{1}(t)$ and $V_{2}(t)$ are expressed as follows:

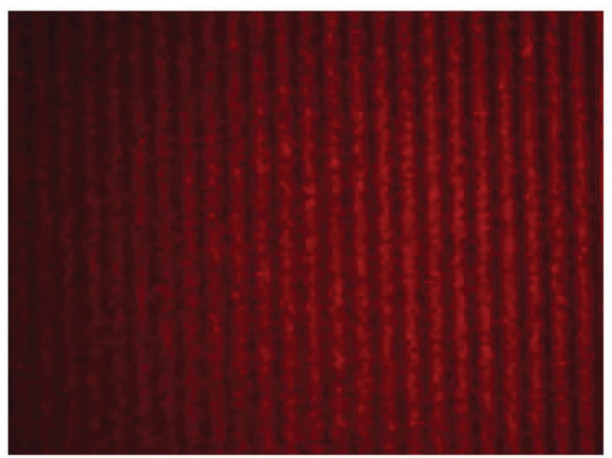

(a) fringes image before the phase stability

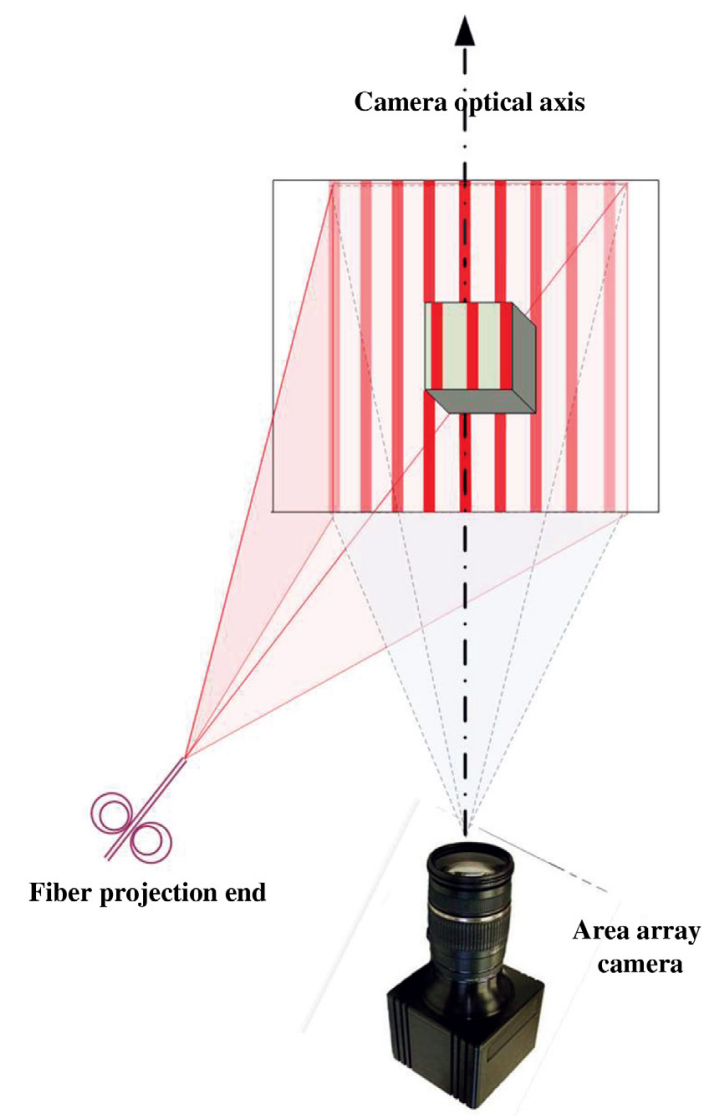

Fig. 9 Schematic of small-object 3-D shape measurement.

$$
\begin{aligned}
V_{3}(t) & =k y \cos \left[\varphi_{0}+\varphi_{s}(t)-\Delta \varphi(t)\right] \\
& =-k y \sin \left[\varphi_{0}+\varphi_{s}(t)-\Delta \varphi(t)-\pi / 2\right]
\end{aligned}
$$

where $k$ is the gain of the differential amplifier and $y$ is the phase voltage conversion factor. $V_{3}(t)$ can be approximated as zero when the phase difference between the two optical fiber arms is at the quadrature state. If the random phase shift $\varphi_{s}(t)$ compels the system to deviate from the quadrature state, $V_{3}(t) \neq 0$; hence, this condition generates a phase error signal $\Delta \varphi_{s}(t)$. The signal $\Delta \varphi_{s}(t)$ with integration operation as a feedback control voltage to drive the

Fig. 8 Interference fringe images captured by an area array camera. (a) Fringe image before the phase stability and (b) fringe image after the phase stability. 
PZT expansion and contraction alters the optical path difference between the two optical interferometer arms to the generate phase shift compensation signal $\Delta \varphi(t)$. Finally, the interferometer system is maintained at the quadrature operating situation. We design the integral feedback circuit to accumulate error and maintain the system steady. The signal $V_{3}(t)$ is integrated to drive the PZT. The interferometer produces the phase shift optical output. The feedback voltage $V_{f}(t)$ is defined as follows:

$V_{f}(t)=g \int_{0}^{t_{0}} V_{3}(t) \mathrm{d} t$

where $g$ is the integral coefficient. The feedback signal $V_{f}(t)$ was applied to the PZT, thereby obtaining the phase change between the two optical interferences at $\Delta \varphi(t)=K V_{f}(t)$. The factors $k, y, g$, and $K$ are adjusted such that the interferometer in the orthogonal state is maintained:
$K V_{f}(t)=\varphi_{s}(t)+\varphi_{0}-\frac{\pi}{2}$

According to this principle, an active phase-tracking homodyne detection method can achieve phase stability and compensation processing for the phase modulation fiber sensing system. Moreover, a precise and real-time phase measurement with high accuracy can be achieved.

The interference fringe images are captured by an area array camera when the temperature fluctuates, as shown in Fig. 8(a). Then, the fringes become fuzzy. However, as shown by Fig. 8(b), the fringe images indicate a high resolution and clear details through the phase stability system. Therefore, the phase stability system is suitable for the accurate 3-D shape measurement of small-object surfaces.

\section{Experimental Results and Analysis}

In accordance with the small-object 3-D shape measurement principle and mathematical model based on optical fiber interference fringe projection, we constructed

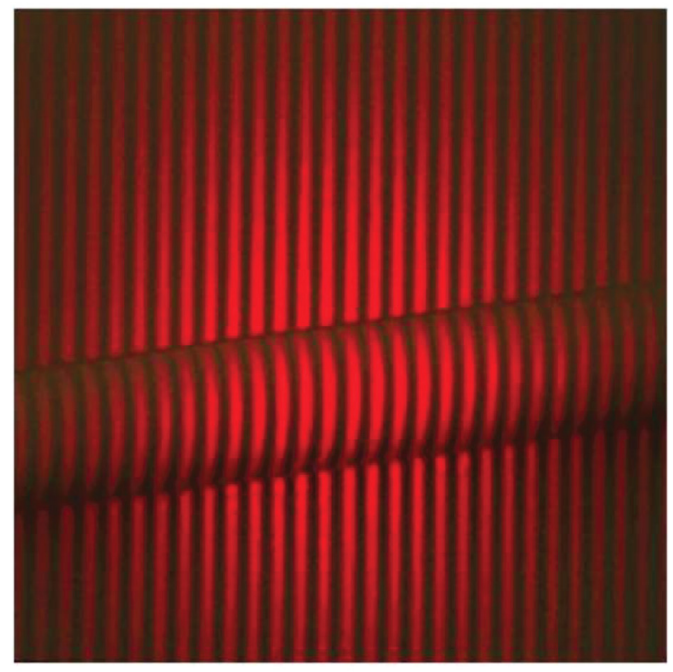

(b) sample 2

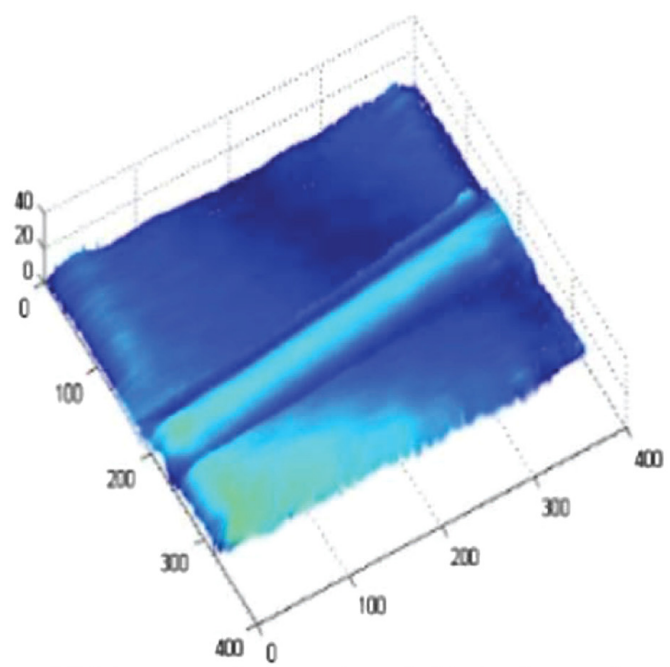

(d) Topography results of (b) (unit: $\mathrm{mm}$ )

(c) Topography results of (a) (unit: mm)

Fig. 10 The result of small object 3-D shape measurement. (a) Circular mold, (b) sample 2, (c) topography results of (a) (unit: $\mathrm{mm}$ ), and (d) topography results of (b) (unit: $\mathrm{mm}$ ). 
a Mach-Zehnder interferometer capable of high-density structure light fringe pattern projections within a small field-of-view range (the field radius was $50 \mathrm{~mm}$ ) and 3-D measurement of small objects. Because we were researching on the measurement technology for the small-object surface 3-D shape, the range of dimensions of the measured objects is 4 to $1000 \mathrm{~mm}^{2}$. Therefore, in order to ensure that the objects can be included in the interference fringes projection area completely, we must obtain high spatial frequency projection fringes in the field of radius $50 \mathrm{~mm}$. We selected the 1101/P-type He-Ne gas laser produced by JDS Uniphase Ltd., whose output power is $1.5 \mathrm{~mW}$, wavelength is $\lambda=632.8 \mathrm{~nm}$, and output light diameter is $d=$ $0.63 \pm 3 \% \mathrm{~mm}$. The fiber used was an F-SV-type singlemode fiber, which operated at a wavelength of $633 \mathrm{~nm}$, core diameter of $4.3 \mu \mathrm{m}$, and maximum attenuation of $15 \mathrm{~dB} / \mathrm{km}$. An F3284 $2 \times 2$-type 3-dB single-mode fiber coupler was used in the system as the spectroscopic equipment. The numerical aperture was 0.13 , which matched the singlemode fiber and coupling lens.

The system of the working principle is shown in Fig. 9. The reference plane was placed in the interference fringe projected field, with a distance to the optical output end of $500 \mathrm{~mm}$ and an interference fringe diameter of $100 \mathrm{~mm}$. The interference fringe images projected on the reference plane exhibit the highest brightness after trimming a precise fiber coupling platform, and the number of interference fringes is $\sim 30$ in the field of radius $50 \mathrm{~mm}$; moreover, the number of fringes superposed on the object requires a change in projection and fiber core distance. The number is related to fringe-phase extraction algorithms because the higher the spatial frequency, the higher is the phase extraction accuracy; therefore, the maximum fringe-number density is the optimum number under the current interferometer hardware conditions. The phase stabilization system steadied the phase of the interference fringes patterns. The resolution of the area array CMOS camera (i-SPEED3 Olympus) was $1280 \times 1024$. The camera was positioned perpendicular to the reference plane. The interference fringes on the reference plane were placed at the center position of the imaging plane of the camera.

In the actual measurements, the interference fringe images on the reference plane were considered as the reference. The measured object in front of the reference plane was fixed. This setup enabled the camera to capture the deformed fringe images modulated by the measured object. The input was viewed on a computer. The enhancement and filtering processes for the deformation striped and reference images were considered, and the phase information of the two interference

Table 1 Measurement results $(\mathrm{mm})$.

\begin{tabular}{lcccc}
\hline $\begin{array}{l}\text { Measuring } \\
\text { object }\end{array}$ & $\begin{array}{c}\text { Actual } \\
\text { height value }\end{array}$ & DLP & $\begin{array}{c}\text { Physical } \\
\text { gratings }\end{array}$ & $\begin{array}{c}\text { Optical } \\
\text { fiber }\end{array}$ \\
\hline Step & 9 & 7.632 & 10.012 & 8.953 \\
Round table & 5 & 6.235 & 6.354 & 5.121 \\
Ramp board & 6.5 & 3.397 & 4.553 & 6.734 \\
Cylinder & 7.5 & 9.245 & 8.932 & 7.236 \\
\hline
\end{tabular}

fringe images was extracted by Fourier transform profilometry and phase unwrapping algorithm. Finally, the height distribution information of the object surface was obtained by using the phase-height mathematical model of the measurement system. The 3-D measurement was obtained from the circular and cylindrical molds through this experimental system. The results are shown in Fig. 10. The accuracy of the measurement system exceeded those of other methods by

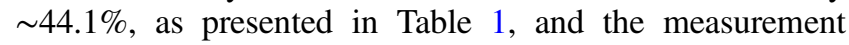
time was shortened by $63.2 \%$. For small-sized components, the root mean square error of the measurement results attained 0.612 , which satisfied the requirements of practical applications.

\section{Conclusion}

In this work, a new method was designed for measuring the 3-D topographies of small objects on the basis of fiber interference fringe projection. The method was validated by constructing a mathematical model in accordance with the 3-D information of objects recovered by the fringe phase information extraction algorithm. This model enabled the height measurement of objects within a $10 \times 10 \mathrm{~cm}$ area, and the measurement system significantly improved the speed and accuracy. The 3-D topography measurement of the small parts was achieved by programming the host computer software, which further verified the feasibility of the 3-D measurement system based on fiber optic interferometric fringe projection.

\section{Acknowledgments}

National Natural Science Foundation of China (NSFC) (Grant Nos. 61405143, 61711530652, 61675154, and 61177078), Tianjin Application Foundation and Frontier Technology Research Plan (Grant No. 14JCYBJC42300), Tianjin Natural Science Foundation (Grant No. 18JCYBJC29100), Tianjin Municipal Education Committee Project (Grant No. TJPUZK20170103), and Sêr Cymru National Research Network in Advanced Engineering and Materials.

\section{References}

1. J. Zhao et al., "Single-camera displacement field correlation method for centrosymmetric 3d dynamic deformation measurement," Meas. Sci. Technot. 29(5), 055401 (2018).

2. M. Patel et al., "Rapid, topology-based particle tracking for high-resolution measurements of large complex 3d motion fields," 5 ci. Rep. 8(1), 5581 (2018).

3. H. Zhao, Y. Xu, and H. Jiang, " $3 \mathrm{~d}$ shape measurement in the presence of strong interreflections by epipolar imaging and regional fringe projection," Opt. Express 26(6), 7117-7131 (2018).

4. V. Srinivasan, H. Liu, and M. Halioua, "Automated phase-measuring profilometry of 3-d diffuse objects," Appl. Opt. 23(18), 3105-3108 (1984).

5. M. Halioua and H. Liu, "Optical three-dimensional sensing by phase measuring profilometry," Opt. Lasers Eng. 11(3), 185-215 (1989).

6. Y. Hung et al., "Practical three-dimensional computer vision techniques for full-field surface measurement," Opt. Eng. 39(1), 143-149 (1999).

7. A. Inanç et al., "3-d optical profilometry at micron scale with multi-frequency fringe projection using modified fiber optic Lloyd's mirror technique," Opt. Lasers Eng. 105, 14-26 (2018).

8. M. Fujigaki, T. Hayashi, and Y. Murata, "Application of phase-shifted fringe projection method with linear fiber arrays using Talbot effect to height measurement of BGA," in Third Int. Conf. on Advances in Optical Science and Engineering, pp. 41-47 (2017).

9. E. Bo et al., "A surface profile reconstruction system using sinusoidal phase-modulating interferometry and fiber-optic fringe projection," Opt. Fiber Technol. 20(3), 294-298 (2014).

10. E. Bo et al., "Sinusoidal phase modulating interferometry system for $3 \mathrm{~d}$ profile measurement," Opt. Laser Technol. 59, 137-142 (2014). 
11. F. Feng et al., "A disturbance-free surface profile measuring system with sinusoidal phase integrating-bucket modulation," Measurement 60, 207-213 (2014)

12. H. Matthews, D. Hamilton, and J. Colin, "Sheppard surface profiling by phase-locked interferometry," Appl. Opt. 25(14), 2372-2374 (1986).

13. C. Mercer and G. Beheim, "Fiber optic phase stepping system for interferometry," Appl. Opt. 30(7), 729-734 (1991).

14. A. Freschi and J. Frejlich, "Adjustable phase control in stabilized interferometry," Opt. Lett. 20(6), 635-637 (1995).

15. A. Kudryashov and A. Seliverstov, "Adaptive stabilized interferometer with laser diode," Opt. Commun. 120, 239-244 (1995).

16. A. Moore et al., "Closed-loop phase stepping in a calibrated fiber-optic fringe projector for shape measurement," Appl. Opt. 41(16), 3348-3354 (2002).
17. D. Wu et al., "Spatial phase-shifting method for adaptive vibrationresistant interferometer," Acta Photonica Sin. 32(8), 969-972 (2003).

Hongqiang $\mathrm{Li}$ is currently a professor in the School of Electronics and Information Engineering at Tianjin Polytechnic University, China, and performs research activity at Tianjin Key Laboratory of Optoelectronic Detection Technology and Systems, China. He has published more than 130 journal and conference papers. His current research interests include fiber Bragg grating sensors, interrogation system, and silicon-based photonic integration.

Biographies for the other authors are not available. 